\title{
Effect of Ambipolar Diffusion on the Flow of a Two-Component Plasma Gas Model in the Earth's Planetary lonosphere
}

\author{
B. S. Tuduo*, T. M. Abbey \\ Email address: \\ bieseb4prom@gmail.com (B. S. Tuduo), tamunoimi.abbey@uniport.edu.ng (T. M. Abbey) \\ ${ }^{*}$ Corresponding author
}

Applied Mathematics and Theoretical Physics Group, Department of Physics, University of Port Harcourt, Port Harcourt, Nigeria

To cite this article:

B. S. Tuduo, T. M. Abbey. Effect of Ambipolar Diffusion on the Flow of a Two-Component Plasma Gas Model in the Earth's Planetary Ionosphere. International Journal of Astrophysics and Space Science. Vol. 5, No. 3, 2017, pp. 47-54. doi: 10.11648/j.ijass.20170503.12

Received: December 23, 2016; Accepted: January 5, 2017; Published: August 22, 2017

\begin{abstract}
The paper presents analytical study on the effect of ambipolar diffusion on the flow of a two-component plasma gas in the Earth's Planetary Ionosphere as a model to examine the ions-neutral and electrons-neutral atom interactions. The problem which consists of a set of partial non-linear differential equations was addressed using a plane wave and perturbation method of solutions. The result indicates that plasma frequency and electron-density in the Ionosphere increase with increase in magnetic field strength as well as with radiation and free convection parameters. It is observed that for; $\frac{\left(M^{2}+G_{r}\right)^{2}}{f_{k n}\left(G_{r}-f_{k n}\right)} \geq 0$, the plasma interactive state becomes more stable, otherwise some bit of oscillation is noticed. The stability is seen to depend on the magnetic $\left(\mathrm{M}^{2}\right)$ and thermal convection $\left(\mathrm{G}_{\mathrm{r}}\right)$ parameters. Under this condition the signal propagation becomes less diffuse when the frequency of the signal is far greater than the plasma frequency, that is, $\omega>>p$. The study aids our understanding of the effect of coupling frequency on the propagation of satellite signals through the ionosphere.
\end{abstract}

Keywords: Ambipolar Diffusion, Two-Component Plasma Flow, Planetary Ionosphere

\section{Introduction}

The study of the ionosphere and its characteristics are important for both practical and scientific purposes; significantly due to its influence on the propagation of electromagnetic waves. Its studies date back to the pioneer work of Gughelmo Marconi, who successfully transmitted radio signal across the transatlantic on the $12^{\text {th }}$ of December, $1901[1,2]$. Today, the understanding of the chemical and dynamical processes of the ionospheric and astrophysical plasma has found applications in the areas of condensed matter Physics, nuclear Physics, thin panel displays (plasma TV), particle accelerators and communication, just to mention but a few. The ionosphere, which is the ionized part of the Earth's upper atmosphere lies between the 60 and 1000 $\mathrm{km}$, is known to be stratified into D, E, F1 and F2 layers respectively [3 - 5]. The solar extreme ultraviolent (EUV) and $\mathrm{x}$-ray radiations as well as other cosmic radiations account for the ionization of the ionosphere [6 - 9], with the

\section{D-layer being the lest ionized.}

Hence the D-layer, similar to the chromosphere of the solar atmosphere [10], is weakly ionized and as such a collision dominated region; collisions between electrons and ions, and neutral gas and the ionized species are frequent. The co-existence of the ionized species and the neutral gas explains the high recombination in the D-layer [11 - 14] as well as in the in the core of molecular clouds. Electrons which are very light moves faster and tend to separate from the more massive ions. This set up a charge imbalance, and the resulting field slows down the electrons and accelerates the ions, such that they both move together with the same velocity, drifting through the plasma as coupled particles, in the process colliding with other electrons, ions and the neutral gas. [1] and [15] show that the relative motion of ions and neutrals in a weakly ionized medium leads to the coupling of the ions and neutrals via mutual collision, and they drift together through the plasma resulting in ambipolar diffusion. According to [16], [17] and [18], ambipolar 
diffusion is prevalent in the core of molecular clouds which is a key factor in star formation (especially in the transfer of mass and momentum [19]), and the transfer and dissipation of energy in the Sun's chromosphere [10].

Several researchers in the literature have extensively used numerical methods in the study of partially ionized plasma within the frame of single- or two-fluid models [20 - 26]. For example, [20] used semi-implicit scheme for two-fluid ambipolar diffusion to investigate instability in steady-state continuous shocks, while [21] described an explicit method for single-fluid ambipolar diffusion in the strong coupling limit. But, [27] investigated the heating through ambipolar diffusion in turbulent molecular clouds using a single-fluid approximation. Numerical schemes for the multi-fluid treatment of hall diffusion and ambipolar diffusion have been suggested by [28 - 30]. Whereas, [31] and [32] studied the properties of turbulence with ambipolar diffusion in a twofluid approximation using three-dimensional (3D) numerical simulations model. Similarly, [26] presented a semi-implicit method for ambipolar diffusion using a two-fluid approximation. A fully explicit method for incorporating the single-fluid ambipolar diffusion into a multi-dimensional magnetohydrodynamic (MHD) code based on the total variation diminishing (TVD) scheme was described by [14]. While, [15] conducted a three-dimensional (3D) local sharing-box simulations to explore the effect of ambipolar diffusion on the non-linear evolution of magnetorotational instability (MRI) in the strong coupling limit. And just recently, [33] studied the dynamics of the interactions of neutrals and charged plasma particles in a single-fluid, ideal magnetohydrodynamic framework to describe the propagation of low-frequency waves in the partially ionized collision-dominated lower Earth's Ionosphere, and the ionosphere-magnetosphere coupling.

In all the above studies, the effect of temperature differentials with accompanied radiative heat transfer and free convection motion; which is paramount in astrophysical regions where high temperature are experienced was not considered. This is the crux of this present study.

The paper is organized in the following format: - In section 2 , the physics and the mathematical formulation of the problem leading to equation of motion shall be presented, while section 3 has the method of analysis leading to the derivation of different wave-modes and the attendant effects on the electrons and ions density variation in the ionosphere. Section 4 on the other hand discusses the various results and section 5 presents the conclusion. The study will aid our understanding of the chemical and dynamical processes of the ionosphere. It would further shed a light on the physics and factors affecting navigational satellites and global positioning systems.

\section{Problem Formulations}

In most regions of the ionosphere, particularly in the Dlayer and some part of E-layer, ionization is seen to be weak. In this case the region is permeated with neutral gas molecules along with ions and electrons. According to [15] in weakly ionized plasma, where ambipolar diffusion occurs as a result of the relative motion of the ions and neutrals, the inertia of the ionized species is usually negligible. It follows, therefore, that the ion velocity can be determined by the balance between the Lorentz force and ion-neutral gas collisional drag. It is this force balance along with other factors that determined and predict the motion of such an interacting system. If, therefore, $\mathrm{V}, \mathrm{B}, \mathrm{P}, g$ and $\rho$ are respectively the velocity field vector, magnetic field vector, the plasma pressure, the gravitational field vector and the density of the plasma component species; while $\mathrm{q}_{\mathrm{r}}$ is the radiation flux resulting from the interaction of the cosmic and X-rays from the solar body on the Earth's ionosphere; $\sigma$, the Stefan Boltzmann constant; $\alpha$, the plasma optical property; $\mathrm{T}$, the temperature of the medium and $\mathrm{K}_{0}, \chi_{k}, \mathrm{C}_{\mathrm{p}}, \mu$ and $e$ are the thermal conductivity, porosity, specific heat capacity at constant pressure, neutral gas viscosity and electron charge, $\mathrm{f}_{k n}$, the coupling frequency between the ionized species and the neutral gas, whereas $\mathrm{J}$ is the current density, then the mathematical statements governing the flow of the twocomponent plasma model in the present of ambipolar diffusion are as follows;

$$
\begin{gathered}
\frac{\partial \rho_{k}}{\partial t}=\mathrm{Q}-\mathrm{L}-\nabla\left(\rho_{k} V_{k}\right) \\
\rho_{k} \frac{\partial V_{k}}{\partial t}=-\nabla P_{k}+\mu \nabla^{2} V_{k}+\rho_{k} g+\frac{e \rho_{k}}{m_{k}}\left(\mathrm{E}+V_{k} x B\right)+ \\
\frac{\mu_{r}}{\chi_{k}} V_{k} \pm \rho_{k} f_{k n}\left(V_{k}-V_{n}\right) \\
\rho_{k} \mathrm{C}_{\mathrm{p}} \frac{\partial T_{k}}{\partial t}=k_{0}^{2} \nabla^{2} T_{k}-\nabla q_{r} \\
\nabla^{2} q_{r}-3 \alpha^{2} q_{r}-16 \sigma \alpha T^{2} \nabla T=0 \\
\nabla \times \mathrm{E}=-\frac{1}{C} \frac{\partial B_{1}}{\partial t}, \\
\nabla \times B_{1}=\frac{1}{C} \frac{\partial E}{\partial t}+\frac{4 \pi}{C} \mathrm{~J} \\
\nabla \cdot \mathrm{E}=4 \pi\left(e^{+} \rho_{i} V_{i}+e^{-} \rho_{e} V_{e}\right) \\
\nabla \cdot B_{1}=0
\end{gathered}
$$

and

$$
\mathrm{J}=\left(e^{+} \rho_{i} V_{i}+e^{-} \rho_{e} V_{e}\right)
$$

where, $(k=i$ or $e)$ denote the ions and electron and $n$ the neutral species respectively, while $Q$ and $L$ are the rate of production of ions from the neutral gas and the rate of loss of ions due to recombination. Here equations (1 to 4) express the flow variables of the two-component plasma model. Equation (1) is the continuity equation, which expresses the mass conservation of electrons, ions and neutral gas (atoms) in the ionosphere and $\nabla\left(\rho_{k} V_{k}\right)$ is the loss of ionization due to transport with $V_{k}$ being the transport velocity. In the absence of $\mathrm{Q}$ and $\mathrm{L}$, that is, the case in which $Q=0$ and $L=0$, the continuity equation assumes the form:

$$
\frac{\partial \rho_{k}}{\partial t}+\nabla \cdot\left(\rho_{k} V_{k}\right)=0
$$


On the other hand, equation (2) shows, the force balance of the plasma within the ionosphere, the term $\nabla P_{k}$ is the force due to the pressure gradient, whereas the present of $\rho_{\mathrm{k}} g, \frac{e \rho_{k}}{m_{k}}$ $\left(E+V_{k} x B\right)$ and $\rho_{k} f_{k n}\left(V_{k}-V_{n}\right)\{k \neq n\}$ terms indicate forces due to gravity, electromagnetic fields and collision (or friction) between the ions $(i)$ and the neutrals $(n)$ with $f_{k n}$ being the collisional frequency.

Also, equation (3) is the energy conservation equation, which indicates the present of $k_{0}^{2} \nabla^{2} T_{k}$, the heat conduction term and $\nabla q_{r}$, the heat absorption and transfer term. As we can readily see from equations (1) to (4), the flow variables are highly coupled and the equations expressing them are non-linear. Therefore, to tackle the problem analytically some useful assumptions are necessary. For example, in the intergalactic, interplanetary regions, stellar atmospheres and in some layers of the ionosphere the gases are seen to be rarefied [10], such that the corresponding optical property, $\alpha$ is far less than one (i.e., $\alpha \ll 1)$. In this case, as was in the case of [34], the integro-differential equation in equation (4) becomes:

$$
\nabla q_{r}=4 \sigma \alpha\left(T^{4}-T_{\infty}^{4}\right)
$$

where, $T_{\infty}$ is the temperature at which the plasma gas is in a state of equilibrium. Also, if we further assume as in the case of $[5,35-38]$, that the temperature difference between adjacent layers of the plasma is not much compared to each other, thus;

$$
T_{k, n}=T_{\infty}+\phi
$$

where, $\phi$ is a small temperature correction factor, such that, $\theta(\mathrm{T}) \gg \phi \gg \theta\left(T_{\infty}\right)$, then, the heat transfer flux vector equation reduced to;

$$
\nabla q_{r}=16 \sigma \alpha \mathrm{T}^{3}\left(\phi+T_{\infty}\right)
$$

This equation shall be substituted into equation (3) and solved along with equations (1) and (2). Equations (5-9) are the Maxwell's equations; where, $B_{1}$ is the induced magnetic field vector and $B=B_{0}+B_{1}$, with $B_{0}$ being the applied magnetic field. Eliminating $B_{1}$ by combining equations (5) and (6) we have the following form of equation:

$$
\nabla \times \nabla \times \mathbf{E}=-\frac{1}{C^{2}} \frac{\partial^{2} E}{\partial t^{2}}-\frac{4 \pi}{C} \frac{\partial J}{\partial t}
$$

This brings the mathematical statement of the problem to conclusion.

\section{Method of Solutions and Analysis}

In a typical plasma setting viscosity, coriolis and centripetal accelerations, and tidal waves are usually neglected for simplicity, thus equation (2) becomes;

$$
\rho_{k} \frac{\partial V_{k}}{\partial t}=-\nabla P_{k}+\rho_{k} g+\frac{\mathrm{e} \rho_{k}}{m_{k}}\left(\mathrm{E}+V_{k} \times \mathrm{B}\right)+\frac{\mu_{r}}{\chi_{k}} V_{k} \pm \rho_{k} \mathrm{f}_{k n}\left(V_{k}-V_{n}\right)
$$

such that, for the ions, we have;

$$
\begin{gathered}
\rho_{i} m_{i} \frac{\partial V_{i}}{\partial t}=-K_{B} T_{i} \nabla \rho_{i}+\rho_{i} m_{i} g+\mathrm{e} \rho_{i}\left(\mathrm{E}+V_{i} \times \mathrm{B}\right)+\frac{\mu_{r}}{\chi_{i}} m_{i} V_{i}+ \\
\rho_{i} m_{i} f_{i n}\left(V_{i}-V_{n}\right)
\end{gathered}
$$

and for the electrons, we have;

$$
\begin{aligned}
\rho_{e} m_{e} \frac{\partial V_{e}}{\partial t}=-K_{B} & T_{e} \nabla \rho_{e}-\rho_{e} m_{e} g-\mathrm{e} \rho_{e}\left(\mathrm{E}+V_{e} \times \mathrm{B}\right)+\frac{\mu_{r}}{\chi_{e}} m_{e} V_{e} \\
& +\rho_{e} m_{e} \mathrm{f}_{e n}\left(V_{e}-V_{n}\right)
\end{aligned}
$$

whereas, for the neutral gas, it becomes;

$$
\rho_{n} m_{n} \frac{\partial V_{n}}{\partial t}=-K_{B} T_{n} \nabla \rho_{n}-\rho_{n} m_{n} g+\frac{\mu_{r}}{\chi_{n}} m_{n} V_{n}+\rho_{n} m_{n} f_{n i}\left(V_{n}-V_{i}\right)
$$

Experimental observation [3] shows that the ion-neutral gas collision frequency $f_{i n}$ or $f_{n i}$ and the electron-neutral gas collision frequency $f_{\text {en }}$ can be given as;

$$
f_{\text {in }}=2.6 \times 10^{-15}\left(\rho_{n}+\rho_{i}\right)\left[M_{n}^{\prime}\right]^{1 / 2}
$$

and

$$
f_{e n}=5.4 \times 10^{-16} \rho_{n} T_{e}^{1 / 2}
$$

where, $M_{n}^{\prime}$ and $T_{e}$ denote the mean neutral molecular mass and electron temperature.

Now, if $\rho_{n}$ and $\rho_{i}, V_{n}$ and $V_{i}$ are respectively the density of neutral gas and ions as well as their velocities. In the absent of every other force the equilibrium motion of the plasma can be determined by the balance between the magnetic field (that is, the Lorentz force) and the ambipolar field which exists as a result of the relative motion between the ions and the neutral gas, thus;

$$
\mathrm{J} \times \mathrm{B}=\gamma \rho_{n} \rho_{\mathrm{i}} \mathrm{m}_{\mathrm{i}}\left(\mathrm{V}_{\mathrm{i}}-\mathrm{V}_{\mathrm{n}}\right)
$$

where, $\gamma=\frac{\langle\sigma v\rangle}{m_{n}+m_{i}}$, with $\langle\sigma v\rangle$ being the momentum transfer rate between the ions and the neutral gas, and $m_{i}$ and $m_{n}$ are the masses of the ions and neutral gas. This indicates, according to $[15,33]$ that the neutral gas gets hook up to the magnetic field through its interactions with the ions, such that the induction equations for the neutrals assumed the forms;

$$
\rho_{n} \frac{\partial V_{n}}{\partial t}=\nabla \times\left(v_{n} \times \mathrm{B}\right)-\frac{4 \pi}{C} \nabla \times\left[\frac{v_{A}^{2}}{\mathrm{\gamma} \rho_{i}} J_{\perp}\right]
$$

where, $v_{A}=\frac{B}{\sqrt{\left(4 \pi \rho_{n}\right)}}$ is the Alfven velocity while $J_{\perp}$ is the component of the current density in the perpendicular direction. The term $\frac{4 \pi}{C} \nabla \times\left[\frac{v_{A}^{2}}{\gamma \rho_{i}} J_{\perp}\right]$ accounts for the ambipolar diffusion, with the ambipolar diffusivity defined as $\eta_{A}=\frac{v_{A}^{2}}{\mathrm{\gamma} \rho_{i}}$.

The effect of ambipolar diffusion on the propagation of plasma waves and satellite signals can be deduced by adopting a spectral analysis and solutions of the form;

$$
f(x, y, t)=F(y) \exp [i(k \cdot x-\omega t)]
$$

for all the flow variables presented in section 2. Where, $F(y)$ is the amplitude of the incident beam propagated in the vertical $y$-direction with the frequency, $\omega$ and $k . x$ expresses 
the phase.

Introducing the following neutrality condition as in $[5,39]$, thus:-

$$
\rho=\rho_{i}+\rho_{e}
$$

and

$$
e^{+} \rho_{i}+e^{-} \rho_{e}=0
$$

Such that the velocity of the centre of gravity which enhance the collective behaviour of the plasma becomes;

$$
\mathrm{U}=\frac{m_{i} \rho_{i} V_{i}+m_{e} \rho_{e} V_{e}}{m_{i} \rho_{i}+m_{e} \rho_{e}}
$$

Eliminating $V_{n}$, the neutral wind velocity in equations (15) and (16), we have;

$$
\frac{\partial}{\partial t}\left(\frac{\partial}{\partial t}+2 \mathrm{f}_{i n}\right) V_{i}=\frac{e^{+}}{m_{i}}\left(\frac{\partial}{\partial t}+\mathrm{f}_{i n}\right)\left[\mathrm{E}+\frac{V_{i} \times \mathrm{B}}{C}\right]+H_{i}
$$

for the ions-neutral gas interaction and,

$$
\frac{\partial}{\partial t}\left(\frac{\partial}{\partial t}+2 \mathrm{f}_{e n}\right) V_{e}=\frac{e^{-}}{m_{e}}\left(\frac{\partial}{\partial t}+\mathrm{f}_{e n}\right)\left[\mathrm{E}+\frac{V_{e} \times \mathrm{B}}{C}\right]+H_{e}
$$

for the electron-neutral gas interaction.

where, $H_{k}=2 g f_{k n}-K_{B} f_{k n}\left(\frac{T_{k}}{m_{k}} \frac{\nabla \rho_{k}}{\rho_{k}}+\frac{T_{n}}{m_{n}} \frac{\nabla \rho_{n}}{\rho_{n}}\right)$;

Also, by multiplying equation (24) with $m_{i} \rho_{i}$ and equation (25) with $m_{e} \rho_{e}$ following the method adopted by [5, 39], and thereafter adding the results considering the case in which, $\mathrm{f}_{\text {in }}=\mathrm{f}_{\text {en }}=\mathrm{f}_{c}$, and in conjunction with equations (23), we have;

$$
\left(\frac{\partial}{\partial t}+2 \mathrm{f}_{c}\right) \frac{\partial U}{\partial t}=\frac{1}{C}\left(\frac{\partial}{\partial t}+\mathrm{f}_{c}\right)(\mathrm{J} \times \mathrm{B})+\left[m_{i} \rho_{i} H_{i}+m_{e} \rho_{e} H_{e}\right]
$$

Similarly, multiplying equation (24) with $\rho_{i} e^{+}$and equation (25) with $\rho_{e} e^{-}$; and adding their results, and then noting that;

$$
\frac{\left(e^{+}\right)^{2} \rho_{i} V_{i}}{m_{i}}+\frac{\left(e^{-}\right)^{2} \rho_{e} V_{e}}{m_{e}}=\left(\frac{e^{+}}{m_{i}}+\frac{e^{-}}{m_{e}}\right) \mathrm{J}-\frac{e^{+} e^{-}}{m_{i} m_{e}}\left(m_{i} \rho_{i}+m_{e} \rho_{e}\right) \mathrm{U}
$$

we have;

$$
\begin{aligned}
& \left(\frac{\partial}{\partial t}+2 \mathrm{f}_{c}\right) \frac{\partial J}{\partial t}=\left[\frac{\left(e^{+}\right)^{2} \rho_{i}}{m_{i}}+\frac{\left(e^{-}\right)^{2} \rho_{e}}{m_{e}}\right]\left(\frac{\partial}{\partial t}+\mathrm{f}_{c}\right) \mathrm{E}+\frac{1}{C}\left(\frac{e^{+}}{m_{i}}+\frac{e^{-}}{m_{e}}\right) \frac{\partial}{\partial t}(\mathrm{~J} \times \mathrm{B}) \\
& -\frac{e^{+} e^{-}}{m_{i} m_{e} C}\left(m_{i} \rho_{i}+m_{e} \rho_{e}\right) \frac{\partial U}{\partial t} \times \mathrm{B}+\mathrm{f}_{c} \frac{1}{C}\left(\frac{e^{+}}{m_{i}}+\frac{e^{-}}{m_{e}}\right)(\mathrm{J} \times \mathrm{B}) \\
& -\mathrm{f}_{c} \frac{e^{+} e^{-}}{m_{i} m_{e} C}\left(m_{i} \rho_{i}+m_{e} \rho_{e}\right)(\mathrm{U} \times \mathrm{B})+e^{+} \rho_{i} H_{i}+e^{-} \rho_{e} H_{e} \\
& {\left[\begin{array}{c}
\left\{( \omega ^ { 4 } + 4 \mathrm { f } _ { c } \omega ^ { 3 } - ( \frac { a _ { 3 } } { c } + 4 \mathrm { f } _ { c } ^ { 2 } ) \omega ^ { 2 } - ( \frac { a _ { 5 } } { c } + \frac { a _ { 3 } } { c } \mathrm { f } _ { c } ) \omega \} \left\{-\mathrm{a}_{1}\left(\omega^{3}-3 \mathrm{f}_{c} \omega^{2}-4 \mathrm{f}_{c}^{2} \omega\right\}\right.\right. \\
\left\{\frac{4 \pi \omega}{c^{2}}\right\}\left\{\frac{\omega^{2}}{c^{2}}-k^{2}\right\}
\end{array}\right]\left[\begin{array}{l}
\mathrm{J} \\
\mathrm{E}
\end{array}\right]=\left[\begin{array}{c}
2 \mathrm{f}_{c}\left(e^{+} \rho_{i} H_{i}+e^{-} \rho_{e} H_{e}\right) \\
0
\end{array}\right]}
\end{aligned}
$$

whose Slater determinant becomes;

$$
\left\|\begin{array}{c}
\left\{( \omega ^ { 4 } + 4 \mathrm { f } _ { c } \omega ^ { 3 } - ( \frac { a _ { 3 } } { C } + 4 \mathrm { f } _ { c } { } ^ { 2 } ) \omega ^ { 2 } - ( \frac { a _ { 5 } } { C } + \frac { a _ { 3 } } { C } \mathrm { f } _ { c } ) \omega \} \left\{-\mathrm{a}_{1}\left(\omega^{3}-3 \mathrm{f}_{c} \omega^{2}-4 \mathrm{f}_{c}{ }^{2} \omega\right\}\right.\right. \\
\left\{\frac{4 \pi \omega}{c^{2}}\right\}\left\{\frac{\omega^{2}}{c^{2}}-k^{2}\right\}
\end{array}\right\|=0
$$

and further analysis of the above equation yields the following frequency spectrum; 


$$
\omega^{6}+b_{4} \omega^{5}+b_{3} \omega^{4}-b_{2} \omega^{3}+b_{1} \omega^{2}+b_{o} \omega=0
$$

where,

$$
\begin{gathered}
b_{0}=c k^{2}\left(a_{5}+a_{3} \mathrm{f}_{c}\right), b_{1}=4 \mathrm{f}_{c} \\
b_{2}=4 \pi \mathrm{a}_{1}-\frac{a_{3}}{c}-4 \mathrm{f}_{c}{ }^{2}-c^{2} k^{2}, b_{3}=12 \pi \mathrm{a}_{1} \mathrm{f}_{c}+\frac{a_{5}}{c}+\frac{a_{3}}{c} \mathrm{f}_{c}+ \\
4 \mathrm{f}_{c} c^{2} k^{2}
\end{gathered}
$$

and $b_{4}=\mathrm{c} k^{2} a_{3}+4 \mathrm{f}_{c}{ }^{2} c^{2} k^{2}-16 \pi \mathrm{a}_{1} \mathrm{f}_{c}{ }^{2}$.

Substituting for $\mathrm{a}_{1}, \mathrm{a}_{3}$ and $\mathrm{a}_{5}$, we have;

$$
\begin{gathered}
b_{0}=2 k^{2}\left[\frac{\rho_{e} e^{+} e^{-}}{m_{e}}+\frac{\rho_{e} e^{+} e^{-}}{m_{i}}\right], b_{1}=4 \mathrm{f}_{c}, \\
b_{3}=3 \mathrm{f}_{c} \omega_{\rho}^{2}+\frac{2 \mathrm{f}_{c}}{c^{2}}\left[\frac{\rho_{e} e^{+} e^{-}}{m_{e}}+\frac{\rho_{e} e^{+} e^{-}}{m_{i}}\right]+4 \mathrm{f}_{c} c^{2} k^{2}, \\
b_{2}=\omega_{\rho}^{2}-\frac{1}{c^{2}}\left[\frac{\rho_{e} e^{+} e^{-}}{m_{e}}+\frac{\rho_{e} e^{+} e^{-}}{m_{i}}\right]-\left(4 \mathrm{f}_{c}{ }^{2}+c^{2} k^{2}\right),
\end{gathered}
$$

and

$$
b_{4}=k^{2}\left[\frac{\rho_{e} e^{+} e^{-}}{m_{e}}+\frac{\rho_{e} e^{+} e^{-}}{m_{i}}\right]-4 \mathrm{f}_{c}{ }^{2} \omega_{\rho}^{2}+4 \mathrm{f}_{c}{ }^{2} c^{2} k^{2} .
$$

where, $\omega_{\rho}^{2}=4 \pi\left[\frac{\left(e^{+}\right)^{2} \rho_{i}}{m_{i}}+\frac{\left(e^{-}\right)^{2} \rho_{e}}{m_{e}}\right] \Rightarrow \omega_{\rho}^{2}=\omega_{\rho_{i}}^{2}+\omega_{\rho_{e}}^{2}$, the plasma frequency

$$
\omega_{\rho_{i}}{ }^{2}=\left[\frac{4 \pi\left(e^{+}\right)^{2} \rho_{i}}{m_{i}}\right], \text { the ion frequency }
$$

and,

$$
\omega_{\rho_{e}}{ }^{2}=\left[\frac{4 \pi\left(e^{-}\right)^{2} \rho_{e}}{m_{e}}\right], \text { the electron frequency }
$$

If we consider the case in which the plasma is bounded by the magnetic field (i.e., $B_{0} \neq 0$ ); it is possible to isolate the following wave modes and their frequencies [5, 39-40];

$$
\begin{array}{ll}
\Omega_{i}=\frac{1}{C}\left[\frac{e^{+} B_{0}}{m_{i}}\right], & \text { (Ion cyclotron frequency) } \\
\Omega_{e}=\frac{1}{C}\left[\frac{e^{-} B_{0}}{m_{e}}\right], & \text { (Electron cyclotron frequency) } \\
\Omega_{m g}=\mu g B_{0}, & \text { (Magneto }- \text { gravity waves ) } \\
\Omega_{g}=g\left[\frac{\rho_{i} e^{+}}{m_{i}}+\frac{\rho_{e} e^{-}}{m_{e}}\right]=g\left(\Omega_{i g}+\Omega_{e g}\right) & (\text { Gravity waves ) } \\
\Omega_{a}=k_{B}\left(\Omega_{i g}+\Omega_{e g}\right) & \text { (Acoustic waves) } \\
\text { and }, & \text { (Magneto - acoustic waves) } \\
\Omega_{m a}=k_{B} B_{0} &
\end{array}
$$

The above spectral analysis, considering equation (30); yields the current density $j$ and the accompanied induced electric field $E$ as:-

$$
j=\frac{2 f_{c}\left(\omega^{2}-k^{2} c^{2}\right)}{c^{2} \Delta}\left[e^{+} \rho_{i} H_{i}+e^{-} \rho_{e} H_{e}\right]
$$

and

$$
E=\frac{8 \pi \omega f_{c}}{c^{2} \Delta}\left[e^{+} \rho_{i} H_{i}+e^{-} \rho_{e} H_{e}\right]
$$

Such that, the ratio $j / E$ becomes;

$$
\delta_{p}=\frac{j}{E}=\frac{\omega^{2}-k^{2} c^{2}}{4 \pi \omega} \Rightarrow \text { Plasma conductivity }
$$

$[5,39]$ show that $\omega^{2}=\omega_{p}^{2}+k^{2} c^{2}$, simplifying the plasma conductivity to;

$$
\delta_{p}=\frac{j}{E}=\frac{\omega_{p}^{2}}{4 \pi \omega}
$$

In the above analysis we have considered the situation in which the temperature of the plasma is constant, that is, assumed to be either the electron temperature, $T_{e}$ and/or ion temperature, $T_{i}$. But in the case where the temperature varies with altitude (i.e., height of the ionosphere) then equation (2) along with equation (12) are considered such that after introducing the following scaling parameters:$\Theta=\frac{T-T_{0}}{T_{k}-T_{0}}, \rho_{k}=\frac{\rho_{k}^{\prime}}{\rho_{0 k}}, \omega=\frac{\omega^{\prime}}{\omega_{k}}, t=\omega_{k} t$ and $v=\frac{v^{\prime}}{c} \quad$ in addition to a few algebraic steps, the ion-neutral collision and electron-neutral collision equations reduced to:

$$
\begin{aligned}
& \left(k^{2}-\frac{1}{4} \omega-M_{1}^{2}\right) V_{i}+\eta^{2} V_{n}=G_{r} \Theta_{i} \\
& \left(k^{2}-\frac{1}{4} \omega-\chi_{1}^{2}\right) V_{n}+\eta^{2} V_{i}=G_{r} \Theta_{n}
\end{aligned}
$$

for the ion-neutral collision and;

$$
\begin{aligned}
& \left(k^{2}-\frac{1}{4} \omega-M_{1}^{2}\right) V_{e}+\eta^{2} V_{n}=G_{r} \Theta_{e} \\
& \left(k^{2}-\frac{1}{4} \omega-\chi_{1}^{2}\right) V_{n}+\eta^{2} V_{e}=G_{r} \Theta_{n}
\end{aligned}
$$

for the electron-neutral collision.

And eliminating $V_{n}$, the neutral gas velocity, we have;

$$
\omega_{i n}^{2}-h_{1} \omega_{i n}+h_{0}=0
$$

and

$$
\omega_{e n}^{2}-h_{1} \omega_{e n}+h_{0}=0
$$

where, $h_{1}=2 k^{2}+\left(\chi_{1}^{2}+M_{1}^{2}+G_{r}\right), h_{0}=k^{4}+\left(\chi_{1}^{2}+M_{1}^{2}+G_{r}\right) k^{2}$ $+\left(M_{1}^{2}+G_{r}\right) \chi_{1}^{2}+\eta^{2} G_{n}-+\eta^{4}$,

$M_{1}^{2}=\left(\chi^{2}+M^{2}+\eta^{2}\right)$ and $\chi_{1}^{2}=\left(\chi^{2}+\eta^{2}\right)$, whereas, $G_{r}$ is the thermal convection parameter, $M^{2}$ is the magnetic parameter, $\eta^{2}$ is the coupling parameter, and $\chi^{2}$ is the porosity.

The amplitude of the velocity can be deduced from equation (2) for a steady state situation, thus;

$$
\left[\rho_{i} m_{i} f_{\text {in }}-\frac{\mu_{r}}{\chi_{c}}\right] V_{i}=\left(\rho_{i} m_{i} f_{i n}\right) V_{n}-K_{B} T_{i} \nabla \rho_{i}+\rho_{i} m_{i} g+\mathrm{e} \rho_{i}\left(\mathrm{E}+V_{i} \mathrm{xB}\right)
$$

for the ions-neutral gas interaction and,

$$
\begin{aligned}
{\left[\rho_{e} m_{e} f_{e n}-\frac{\mu}{\chi_{c}}\right] V_{e}=} & \left(\rho_{e} m_{e} f_{e n}\right) V_{n}-K_{B} T_{e} \nabla \rho_{e}+\rho_{e} m_{e} g- \\
& \mathrm{e} \rho_{e}\left(\mathrm{E}+V_{e} \times \mathrm{B}\right)
\end{aligned}
$$


for the electron-neutral interaction, such that;

$$
\begin{aligned}
& V_{i}=\beta^{*} \rho_{i} m_{i} f_{i n} V_{n}+\beta^{*}\left[\rho_{i} m_{i} g-k_{B} T_{i} \nabla \rho_{i}\right]+\beta^{*} e^{+} \rho_{i}\left[E+V_{i} \times B\right] \\
& V_{e}=\beta^{*} \rho_{e} m_{e} f_{e n} V_{n}+\beta^{*}\left[\rho_{e} m_{e} g-k_{B} T_{e} \nabla \rho_{e}\right]+\beta^{*} e^{-} \rho_{e}\left[E+V_{e} \times B\right] \\
& \text { where, } \beta^{*}=\frac{1}{\left(\rho_{k} m_{k} f_{k n}-\frac{\mu_{r}}{\chi_{c}}\right)}
\end{aligned}
$$

According to [3], the vector $[E+V \times B]$ an be expanded, thus;

$$
\beta^{*} e^{k} \rho_{k}[E+V \times B]=\frac{1}{1+\left(\eta_{k}^{*}\right)^{2}}\left[\frac{\eta^{*}}{B} E\right]+\left[\left(\frac{\eta^{*}}{B}\right)^{2}-\left(\frac{\eta^{*}}{B}\right)^{3}\right]\{[(E+V \times B) \cdot B] \times B\}
$$

For $\eta^{*}<<1$, that $O\left(\eta^{* 2}\right) \rightarrow 0$, which is the case for a weakly ionized plasma, as is in the D-layer of the ionosphere. In this case the velocity amplitude becomes;

$$
V_{k}=\beta_{k}^{*} \rho_{k} m_{k} f_{k n} V_{n}+\beta_{k}^{*}\left[\rho_{k} m_{k} g-k_{B} T_{k} \nabla \rho_{k}\right]+\eta_{k}^{*} \frac{E}{B}
$$

such that

$$
V_{k}=\beta_{k}^{*} \rho_{k} m_{k} f_{k n} V_{n}+\beta_{k}^{*} \rho_{k} k_{B} T_{k}\left[\frac{\rho_{k} m_{k} g}{k_{B} T_{k}}-\frac{\nabla \rho_{k}}{\rho_{k}}\right]+\eta_{k}^{*} \frac{E}{B}
$$

and further simplification yields;

$$
V_{k}-\beta_{k}^{*} \rho_{k} m_{k} f_{k n} V_{n}=\beta_{k}^{*} \rho_{k} k_{B} T_{k}\left[\frac{g}{H_{k}}-\frac{\nabla \rho_{k}}{\rho_{k}}\right]
$$

In the absence of porosity, that is, the permeability of the medium is zero, the results agree with those of [3], thus;

$$
V_{k}=V_{n}+G_{k}+\eta_{k} \frac{E}{B}
$$

and the ambipolar velocity for the ions and electron becomes;

$$
V_{i}-V_{n}=G_{i}+\eta_{i} \frac{E}{B}
$$

and

$$
V_{e}-V_{n}=G_{e}+\eta_{e} \frac{E}{B}
$$

where, $G_{k}=D_{k}\left[\frac{g}{H_{k}}-\frac{\nabla \rho_{k}}{\rho_{k}}\right], D_{k}=\frac{k_{B} T_{k}}{m_{k} f_{k n}}$ is the diffusion coefficient of the ions or electrons as the case may be and $H_{k}=\frac{k_{B} T_{k}}{m_{k}}$ is the scale height of the respective species and $\eta_{k}=\frac{\Omega_{k}}{m_{k} f_{k n}}$ with $\Omega_{k}$ being the gyro-frequency of the respective species.

\section{Results and Discussion}

The plasma medium of the Earth's planetary ionosphere is highly coupled due to the interactions of plasma species as they move with their respective thermal velocities [15, 33, 41]. The electrons have higher thermal velocity therefore; tend to move further away from the comparatively massive ions and in the process set up a polarization current as a result of charge imbalance which drags the ions along with the electrons. This results in ambipolar diffusion as the coupled species move through the plasma interacting with the neutral gas, and electric and magnetic fields in the terrestrial environment $[3,15]$. The velocity of these coupled species as a function of collision frequency is shown in figure 1 . The profile shows that the electron diffusion velocity decreases as the coupling frequency increases.

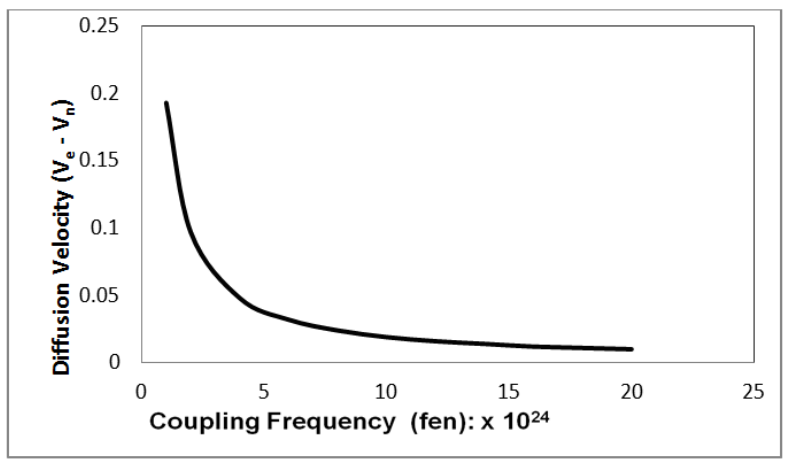

Figure 1. Variation of Diffusion Velocity with Coupling Frequency.

The increase in coupling frequency reduces the diffusivity of plasma medium; therefore, ambipolar diffusion of the coupled species is dampened [42], due to high collision. But, when the plasma temperature is increased the diffusivity is enhanced, due to the fact that the plasma species attain higher thermal velocities. The study further shows that these phenomena affect the propagation of electromagnetic signal via the ionosphere as a result of the interactions of the coupled species in the plasma medium. Furthermore, the coupled plasma species is seen to oscillate in response to the external field as the electromagnetic signal traverse the ionosphere. The interaction of the signal with the coupled species reduces the velocity amplitude of the signal, leading to loss of signal strength with time. The result of this is either the signal is totally lost (absorbed), refracted or partially transmitted, depending on the frequency of propagation in relation to the plasma frequency. [5] Shows that for any signal frequency $(\omega)$ much larger than the plasma frequency (that is, $\omega>>\omega_{\mathrm{p}}$ ) the propagated signal suffer less diffusion. In this case the effect of ambipolar diffusion would not be significant. On the other hand for a case in which $\omega<<\omega_{\mathrm{p}}$, ambipolar diffusion effect is highly noticeable, leading to reduction or loss of signal strength [33]. This is mostly the case in the D-region of the ionosphere, where ionization is weak. Figure 2 illustrates the behaviour of the diffusion velocity with changes in the E/B ratio for large and small coupling frequency ranges. 


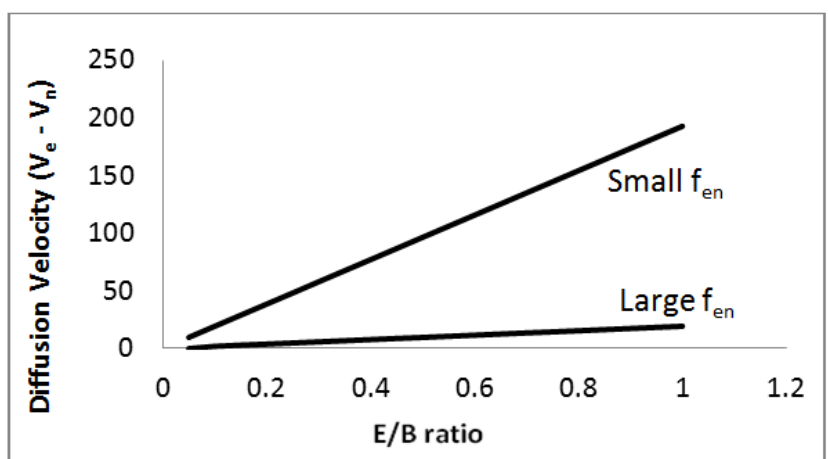

Figure 2. Variation of Diffusion Velocity with Electric-Magnetic Field Ratio.

The curve for the small coupling frequency indicates a much larger velocity growth rate than that for large coupling frequency as the E/B ratio increases. Further analysis shows that when the $\mathrm{E} / \mathrm{B}$ ratio is much greater than one (i.e. $\mathrm{E} / \mathrm{B}$ $>1$, which implies that $\mathrm{B} \rightarrow 0$, or that $\mathrm{B}$ is along $\mathrm{E}$ direction, or that the magnetic field component is negligible compared to the electric field); the ionized species oscillates along the direction of the electric field and thereby leading to increase in the diffusion velocity in the vertical direction. But, when the ratio is far less than one (i.e., $\mathrm{E} / \mathrm{B}<<1$ ) the magnetic field component become much larger than the electric field; therefore, the species moves along the electric field in the perpendicular direction of the magnetic field, the Lorentz forces act on them setting up a torque such that they gyrate in response to the magnetic flux. This results in a reduction of diffusion rate. The flow of plasma across magnetic field lines depends on the ratio of the coupling frequency, $f_{k n}$, to the gyro-frequency, $\Omega_{\mathrm{k}}$ [43]. The analysis indicates that for $\mathrm{f}_{\mathrm{kn}}>\Omega_{\mathrm{k}}$, the collision prevents the plasma from gyrating along the magnetic field lines, instead they move in the electric field direction. But, for $f_{k n}<\Omega_{k}$, the plasma species gyrates along the magnetic field lines. The variation of coupling frequency, $f_{k n}$ as the electron density increases for different wave numbers at constant magnetic strength $\left(\mathrm{M}^{2}=0.5\right)$ is shown in figure 3 .

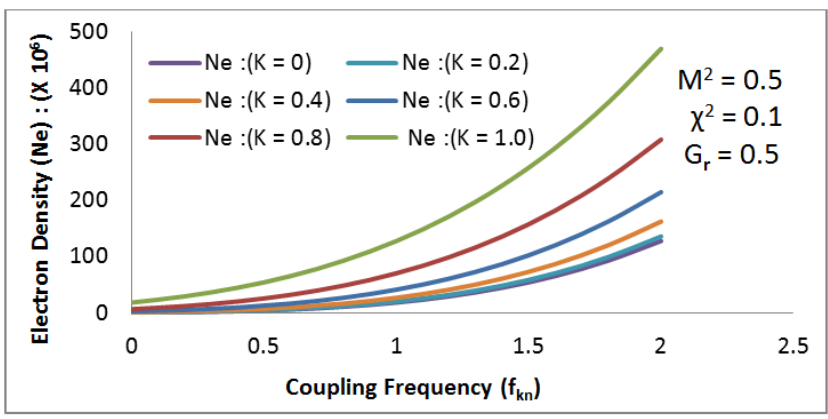

Figure 3. Variation of Electron Density against Coupling Frequency.

Similarly, the profiles presented in figures (4) and (5) indicate that the plasma conductivity $\left(\delta_{p}\right)$ increases with electron density as well as with the height of the ionosphere. At the peak electron density which is at about $450 \mathrm{~km}$ up into the ionosphere, the conductivity attains maximum value and tappers off as the ionization density reduces. These findings are in agreement with those of $[3,43]$.

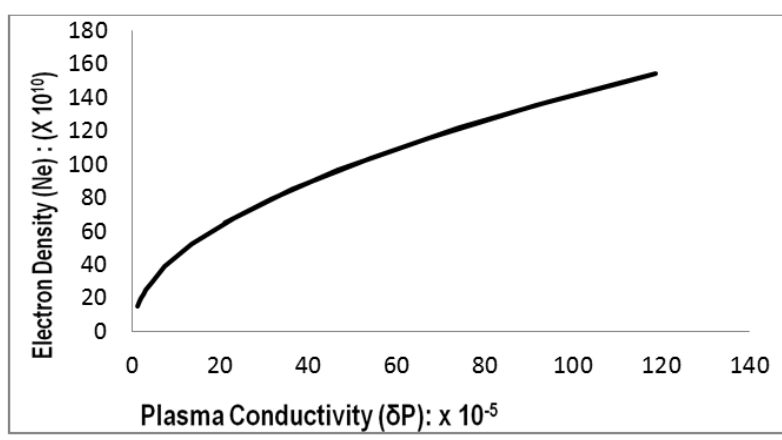

Figure 4. Variation of Plasma Conductivity with Electron Density.

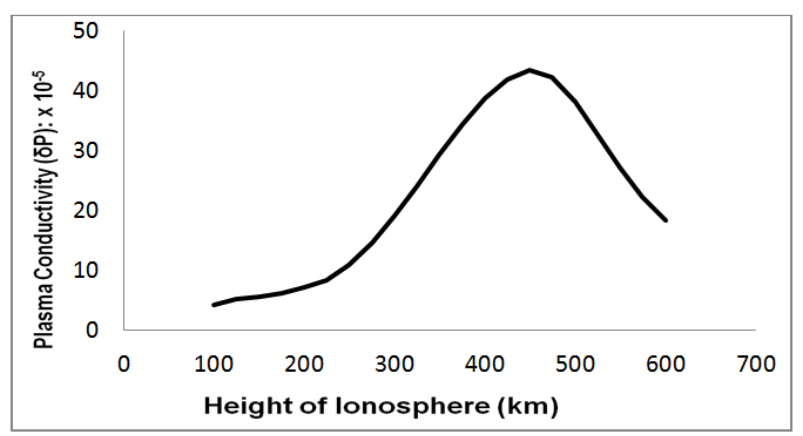

Figure 5. Variation of Plasma Conductivity with Height of Ionosphere.

On the whole, the general analysis shows that when $\frac{\left(M^{2}+G_{r}\right)^{2}}{f_{k n}\left(G_{r}-f_{k n}\right)} \geq 0$, the plasma interactive state becomes more stable, otherwise some bit of oscillation is noticed. The stability is seen to depend among others on the magnetic field strength $\left(M^{2}\right)$ and thermal convection $\left(G_{r}\right)$. Under this condition the signal propagation becomes less diffuse when the frequency is far greater than the plasma frequency, that is, $\omega>>\omega_{\mathrm{p}}$.

The overall analysis presented in this work aids our understanding of the physics of signal and satellite propagation in the present of ambipolar diffusion.

\section{Conclusion}

Ambipolar diffusion, which results from the interactions in a weakly ionized plasma, has been found to have a profound effect on the interactive state and the flow structure of ionospheric plasma. The stability condition observed in the study, aside from shedding a light on the physics and ionospheric plasma interactions, will serve as a veritable tool in mitigating factors affecting signal propagation in navigational satellites and global positioning systems.

\section{References}

[1] Carozzi, T. D. (2000); Radio Waves in the Ionosphere Propagation, generation and detection, IRF Scientific Report 272.

[2] Hunsucker, R. D. and Hargreaves, J. K. (2003); The highlatitude Ionosphere and its efects on radio propagation, Cambridge University Press. 
[3] Balan, N. (2007); Planentary Ionosphere, Kodaikanal Obseratory, Indian Institute of Astrophysics.

[4] Onwuneme, S. E. (2009); F-Layer peak electron density variations in the ionosphere, African J. Phys, 2, 45-57.

[5] Abbey, T. M., Alagoa, K. D. and Onwuneme, S. E., (2011); Propagation of MHD waves in stratified electron-plasma of the ionosphere, African J. Phys. 3, 103-119.

[6] Zolesi, B. and Cander, L. R. (2014); Ionospheric Prediction and Forecasting, Springer Geophysics, (C) Springer-Verlag Berlin Heidelberg.

[7] Kane, R. P. (1992); Sunspots, solar radio noise, solar EUV and Ionospheric fo2, J. Atmos. Terr. Phys., 54, 463-466.

[8] Balan, N., Bailey, G. J., Jenkins, B., Rao, P. B. and Moffett, R. J. (1994); Variations of ionospheric ionization and related solar fluxes during an intense solar cycle, J. Geophys. Res., 99(A2), 2243-2253.

[9] Balan, N., Bailey, G. J. and Moffett, R. J. (1994); Modeling studies of ionospheric variations during an intense solar cycle, J. Geophys. Res., 99(A9), 17467-17475.

[10] Leake, J. E., DeVore, C. R., Thayer, J. P., Burns, A. G., Crowley, G., Gilbert, H. R., Huba, J. D., Krall, J., Linton, M. G., Lukin, V. S., Wang, W., (2016); Chromosphere and Earth's Ionosphere/Thermosphere, arXiv:1310.0405v4 [astro-ph.SR].

[11] Elmegreen, B. G. (1979); ApJ, 232, 729.

[12] Myers, P. C. (1985); Protostars and Planets II, ed. M. S. Matthews and D. C. Black (Tucson, AZ: Univ. Arizona Press), 81 .

[13] Van Loo S., FalleS. A. E. G., Hartquist1, T. W. and Barker, A. J., (2008); The effect of ambipolar resistivity on the formation of dense cores, Astronomy and Astrophysics manuscript no. version 2 .

[14] Choi Eunwoo, Jongsoo Kim and Paul J. Wiita (2009); An explicit scheme for incorporating ambipolar diffusion in a magnetohydrodynamics code, The Astrophysical Journal Supplement Series181, 413.

[15] Bai, Xue-Ning and Stone, M. James (2011); Effect of ambipolar diffusion on the non-linear evolution of Magnetorotational Instability in weakly ionized disks, Princeton University, Princeton, NJ, 08544.

[16] Mestel, L. and Spitzer, L., Jr. (1956); MNRAS, 116, 503.

[17] Mouschovias, T. Ch. (1976); ApJ, 207, 141.

[18] Shu, F. H., Adams, F. C., and Lizano, S. (1987); Ara, A25, 23.

[19] Committee on Solar and Space Physics, National Research Council (2004); Plasma Physics of the Local Cosmos, The National Academies Press, Washington, D. C.

[20] Tóth, G. (1994); ApJ, 425, 171.

[21] Mac Low, M.-M., Norman, M. L., Königl, A., and Wardle, M. (1995); ApJ, 442, 726.
[22] Mac Low, M.-M. and Smith, M. D. (1997); ApJ, 491, 596.

[23] Smith, M. D. and Mac Low, M.-M. (1997); AandA, 326, 801.

[24] Stone, J. M. (1997); ApJ, 487, 271.

[25] Li, P. S., McKee, C. F., and Klein, R. I. (2006); ApJ, 653, 1280 .

[26] Tilley, D. A. and Balsara, D. S. (2008); MNRAS, 389, 1058.

[27] Padoan, P., Zweibel, E., and Nordlund, A. (2000); ApJ, 540, 332.

[28] Falle, S. A. E. G. (2003); MNRAS, 344, 1210.

[29] O'Sullivan, S. and Downes, T. P. (2006); MNRAS, 366, 1329.

[30] O'Sullivan, S. and Downes, T. P. (2007); MNRAS, 376, 1648.

[31] Oishi, J. S. and Mac Low, M.-M. (2006); ApJ, 638, 281.

[32] Li, P. S., McKee, C. F., Klein, R. I., and Fisher, R. T. (2008); ApJ, 684, 380.

[33] Pandey, B. P. (2016); The magnetohydrodynamic description of Earth's ionosphere, arXiv: 1610.03735v1 [physics.plasm$\mathrm{ph}$.

[34] Cheng, P. (1964); Two-Dimensional radiating gas flow by moment method, AIAAJ 21662.

[35] Bestman A. R. (1983); Low Reynolds number flow in a heated tube of varying section, J. Austral. Math. Soc. Ser. B. $25,244-260$.

[36] Abbey, T. M, Bestman, A. R. and Mbeledeogu, I. U. (1992); Flow of a Two-component plasma model in a porous rotating hot sphere, Astrophys and Space Sci, 197, 67-76.

[37] Abbey, T. M. and Bestman, A. R. (1995); Slip flow in a twocomponent plasma model with radiative heat transfer, International Journal of Energy Research, 19: 1-6.

[38] Alagoa, K. D., Tay, G. and Abbey, T. M. (1999); Radiative and convective effects of A MHD flow through a porous medium sandwiched between two infiniteparallel plates with time dependent suction, Astrophysics and space Science, 260,455-468.

[39] Sanderson, J. J. (1974); Plasma waves in plasma physics lecture series, Edited by B. E. Keen, Institute of physics (IOP) - Publishers London.

[40] Priest, E. R. (1982); Solar magneto-hydrodynamics 1, D. Reidel Publishing Company, Holland.

[41] Steven, J. Schwartz, Christopher, J. Owen1, and David Burgess (2002); Astrophysical Plasmas, Astronomy Unit, Queen Mary, University of London, London E1 4NS, U.K.

[42] Desch, S. J. (2004); Linear Analysis of the Magnetorotational Instability including ambipolar diffusion with application to protoplanetary disks, The Astrophysical Journal, 608: 509525.

[43] Stefan, M. (2006); Conductivity of the ionosphere, CIRES, University of Colorado. 